\section{Cardiovascular \\ Disease in Women}

What Is New in the 2013 ACC/AHA Guideline

\author{
on the Treatment of Blood Cholesterol \\ to Reduce Atherosclerotic Risk in Adults?
}

Salim S. Virani, MD, PhD

\section{$\star$ CME Credit}

Presented at the 4th Annual Symposium on Risk, Diagnosis and Treatment of Cardiovascular Disease in Women; Texas Heart Institute, Houston; 19 October 2013.

Section Editor:

Stephanie A. Coulter, MD

Key words: Arterial disease, peripheral; atherosclerosis; cholesterol, low-density-lipoprotein; cholesterol, non-high-density-lipoprotein; guidelines, cholesterol-management; hypercholesterolemia; risk calculator; statin therapy, long-term; statins, use of

From: Health Policy, Quality \& Informatics Program, Michael E. DeBakey VA Medical Center Health Services Research and Development Center for Innovations; Section of Cardiovascular Research, Department of Medicine, Baylor College of Medicine; and Center for Cardiovascular Disease Prevention, Methodist DeBakey Heart \& Vascular Center; Houston, Texas 77030

Address for reprints: Salim S. Virani, MD, PhD, Health Services Research and Development (152), Michael E. DeBakey Veterans Affairs Medical Center 2002 Holcombe Blvd., Houston, TX 77030

E-mail: virani@bcm.edu

(C) 2014 by the Texas Heart ${ }^{\circledR}$ Institute, Houston
$\mathbf{T}$ he American College of Cardiology (ACC) and the American Heart Association (AHA) recently published, in collaboration with the National Heart, Lung, and Blood Institute (NHLBI), the long-awaited 2013 revision of the cholesterol-management guidelines. ${ }^{1}$ The process started in 2008, when an expert panel convened by the NHLBI developed critical questions (CQs), interpreted the evidence, and developed recommendations on the basis of that evidence. In response to a 2011 report from the Institute of Medicine, ${ }^{2}$ the NHLBI Advisory Council recommended that the NHLBI focus on the generation of high-quality evidence and enter into partnerships with other professional organizations to develop practice recommendations. ${ }^{3}$ The recently published ACC/AHA guideline on the treatment of blood cholesterol is the product of this collaboration between the NHLBI and the ACC/ AHA Task Force on Practice Guidelines.

This guideline document differs from the prior Adult Treatment Panel (ATP) III cholesterol-management guidelines. ${ }^{4}$ Unlike earlier cholesterol guidelines, which attempted to encompass the management of all cholesterol parameters, the current document does not intend an exhaustive review. Rather, this guideline document addresses itself to the specific CQs that the expert panel considered most important. Simply put, these guidelines provide more "depth" in answering a small number of basic clinical questions by discussing them in great detail. They are not, therefore, intended as an exhaustive resource for lipid management, but as practice-management guidelines to answering very specific clinical questions.

\section{The 3 Critical Questions Examined by These Cholesterol-Management Guidelines}

The expert panel reviewed the evidence (or lack thereof) for 3 CQs:

CQ1: What is the evidence for low-density-lipoprotein cholesterol (LDL-C) and non-high-density-lipoprotein cholesterol (non-HDL-C) goals for the secondary prevention of atherosclerotic cardiovascular disease (ASCVD)? Atherosclerotic cardiovascular disease includes coronary heart disease (CHD), stroke, and peripheral arterial disease, all of presumed atherosclerotic origin.

CQ2: What is the evidence for LDL-C and non-HDL-C treatment goals for the primary prevention of ASCVD?

CQ3: For primary and secondary prevention, what is the impact on lipid levels, effectiveness, and safety of specific cholesterol-modifying drugs used for lipid management in general and selected subgroups?

Given the fact that almost all the statin trials used a fixed-dose statin, titration to a specific LDL-C or non-HDL-C goal was not performed, and no randomized control trial evaluated titration of all individuals in a treatment group to specific LDL-C (or non-HDL-C) target or compared any two LDL-C (or non-HDL-C) targets; the

Dr. Virani is supported by a Department of Veterans Affairs Health Services Research and Development Service Career Development Award (CDA 09-028). The views expressed in this article are those of the author and do not necessarily represent the views of the Department of Veterans Affairs. 
panel made no recommendation for or against specific LDL-C or non-HDL-C targets for primary or secondary prevention of ASCVD (CQ1, CQ2). Furthermore, most of the treatment recommendations encourage the use of statins as the first-line drug because other lipid-lowering agents have not improved cardiovascular outcomes over and above statin therapy alone. These guidelines, then, have moved away from a cholesterol treat-to-target approach to a statin dose-based approach.

\section{Summary of Recommendations}

These guidelines are intended for application to adults older than 21 years. For purposes of these recommendations, atorvastatin 40 to $80 \mathrm{mg}$ and rosuvastatin 20 to $40 \mathrm{mg}$ are referred to as high-intensity statin therapy (the daily dose lowers LDL-C by approximately $\geq 50 \%$ ), whereas atorvastatin 10 to $20 \mathrm{mg}$, rosuvastatin 5 to 10 $\mathrm{mg}$, simvastatin 20 to $40 \mathrm{mg}$, pravastatin 40 to $80 \mathrm{mg}$, lovastatin $40 \mathrm{mg}$, fluvastatin $80 \mathrm{mg}$, and pitavastatin 2 to $4 \mathrm{mg}$ are referred to as moderate-intensity statin therapy (the daily dose lowers LDL-C by approximately $30 \%$ to $<50 \%$ ). All other statin doses (the daily dose lowers LDL-C by $<30 \%$ ) are considered low-intensity statin therapy. The guideline identifies 4 groups of patients who derive the most benefit from statin therapy:

1) Individuals with established clinical ASCVD, defined as $C H D$, strokes, or peripheral arterial disease of atherosclerotic origin. These patients should receive high-intensity statin therapy, or moderate-intensity statin therapy if they are older than 75 years or otherwise unable to tolerate high-intensity statin therapy.

2) Individuals with primary elevation of $L D L-C \geq 190$ $m g / d L$, who probably have familial hypercholesterolemia. These patients should receive high-intensity statin therapy, or moderate-intensity statin therapy if they are not candidates for high-intensity therapy.

3) Individuals 40 to 75 years of age with diabetes mellitus (type 1 or 2) and with LDL-C levels between 70 and $189 \mathrm{mg} / \mathrm{dL}$. These patients should receive moderate-intensity statin therapy, or high-intensity statin therapy if their estimated 10 -year ASCVD risk is $7.5 \%$ or greater.

4) Individuals without ASCVD or diabetes mellitus who are 40 to 75 years of age and whose estimated 10year ASCVD risk is $\geq 7.5 \%$. These patients should receive moderate- to high-intensity statin therapy.

For groups 3 and 4 above, the 10 -year risk for ASCVD events can be calculated by using the NHLBI pooledcohort equations. A web-based calculator is available online, as is a downloadable spreadsheet that enables the estimation of 10-year and lifetime risk for ASCVD. 5 These equations differ from the ATP III CHD 10-year risk calculator ${ }^{6}$ in several important ways. First, this risk calculator is sex- and race-specific, unlike the prior ATP III risk calculator. Of greater importance is the fact that outcomes estimated by this risk calculator include global cardiovascular disease outcomes (nonfatal myocardial infarction, CHD death, and nonfatal and fatal stroke), whereas the prior ATP III risk calculator estimated only $\mathrm{CHD}$ death and nonfatal myocardial infarction. The guidelines recommend that this risk calculator be used every 4 to 6 years in individuals aged 40 to 79 years without established ASCVD. Last, the guideline identifies several groups of adults with LDL-C $<190 \mathrm{mg} /$ $\mathrm{dL}$ who would not otherwise be candidates for statin therapy, in whom additional factors (such as drug-drug interactions, patient preferences for treatment, and adverse events associated with statin therapy) can inform clinical decision-making. These groups include people with LDL-C $\geq 160 \mathrm{mg} / \mathrm{dL}$ or other evidence of genetic hyperlipidemia, a family history of premature ASCVD (onset $<55$ years in a first-degree male relative or $<65$ years in a first-degree female relative), a high-sensitivity C-reactive protein level of $>2 \mathrm{mg} / \mathrm{L}$, or a coronary artery calcium score of $\geq 300$ Agatston units. Also included are people whose coronary artery calcium scores fall into the $\geq 75$ th percentile for age, sex, and ethnicity, have an ankle-brachial index $<0.9$, or have an elevated lifetime risk of ASCVD.

In conclusion, this guideline document aims to simplify cholesterol management through the identification of specific patient subgroups that would derive benefit from statin therapy (established ASCVD, an LDL-C level of $\geq 190 \mathrm{mg} / \mathrm{dL}$, or a history of type 1 or 2 diabetes) and then refines risk assessment for the rest of the population by the use of a global CVD risk calculator.

\section{References}

1. Stone NJ, Robinson J, Lichtenstein AH, Bairey Merz CN, Lloyd-Jones DM, Blum CB, et al. 2013 ACC/AHA guideline on the treatment of blood cholesterol to reduce atherosclerotic cardiovascular risk in adults: a report of the American College of Cardiology/American Heart Association Task Force on Practice Guidelines. J Am Coll Cardiol 2013 Nov 7. doi: 10.1016/j/jacc.2013.11.002. [Epub ahead of print]

2. National Research Council. Clinical practice guidelines we can trust. Washington (DC): The National Academies Press; 2011. Available from: http://www.nap.edu/catalog. php?record_id=13058 [cited 2014 Apr 2].

3. Gibbons GH, Shurin SB, Mensah GA, Lauer MS. Refocusing the agenda on cardiovascular guidelines: an announcement from the National Heart, Lung, and Blood Institute. J Am Coll Cardiol 2013;62(15):1396-8.

4. Expert Panel on Detection, Evaluation, and Treatment of High Blood Cholesterol in Adults. Executive summary of the third report of the National Cholesterol Education Program (NCEP) Expert Panel on Detection, Evaluation, and Treatment of High Blood Cholesterol in Adults (Adult Treatment Panel III). JAMA 2001;285(19):2486-97.

5. American Heart Association/American College of Cardiology. 2013 prevention guidelines tools: CV risk calculator. Available from: http://my.americanheart.org/cvriskcalculator [cited 2013 Dec 1].

6. National Heart, Lung, and Blood Institute. Risk assessment tool for estimating your 10-year risk of having a heart attack. Available from: http://cvdrisk.nhlbi.nih.gov/calculator.asp [2013 May; cited 2013 Dec 1]. 